Review Article

\title{
Advances in Thermoelectric Materials and Devices for Energy Harnessing and Utilization
}

\author{
KANISHKA BISWAS* \\ New Chemistry Unit, Jawaharlal Nehru Centre for Advanced Scientific Research (JNCASR), Jakkur \\ P.O., Bangalore 560 064, India
}

(Received on 30 March 2014; Accepted on 08 August 2015)

\begin{abstract}
The emerging global need for energy generation, conservation, and utilization has intensified interest in more efficient, costeffective and pollution-free means of power generation. Thermoelectric materials can generate electrical energy from waste heat and can play an important role in a global alternative energy solution. Recently, there have been significant advances in direct thermal-to-electrical energy conversion materials development and this has generated increased interest in the field of chemistry, physics and materials engineering. This article highlights a combination of new high performance materials, new device concepts and future directions to improve material efficiencies and cost effectiveness. Here, I have first presented a brief introduction to thermoelectrics followed by the reason for the importance of the research and development in this field for India. I have discussed about the main criteria for good thermoelectric materials and provided important examples of state-of-the-art materials followed by future direction and conclusions.
\end{abstract}

Keywords: Energy Conversion; Waste Heat Recovery; Thermoelectrics; Thermal Conductivity; Seebeck Coefficient; Earth Abundant Materials

\section{Introduction}

Driven by the demand for clean and sustainable energy sources, thermoelectricity has become a significant part of research portfolio seeking to identify new and efficient energy materials for power generation (Snyder and Toberer, 2008; Sootsman et al., 2009; Chen et al., 2003). Thermoelectric materials can directly and reversibly convert heat energy into electrical energy (Snyder and Toberer, 2008; Sootsman et al., 2009; Chen et al., 2003). The heat can be generated from the combustion of fossil fuels, from sunlight, or as a byproduct of various processes (e.g. combustion of coal and petroleum, chemical reactions, nuclear decay, etc.). Therefore, thermoelectric materials can play a pivotal role in both primary power generation and energy conservation (i.e., waste-heat harvesting). An important topic of discussion is how big this role is likely to be and the answer to this

\footnotetext{
*Author for Correspondence: E-mail: kanishka@jncasr.ac.in
}

question depends exclusively on how efficient these materials are. It is hoped and expected however that thermoelectrics will play a more increasing role than it has in the past and will be one of the several technologies working together to address energy efficiency issues.

Thermoelectric modules are solid-state devices that directly convert thermal energy into electrical energy (Fig. 1). This process is based on the Seebeck effect, which is the appearance of an electrical voltage causes by a temperature gradient across a material. The inverse of this, that is the appearance of a temperature gradient upon the application of voltage is known as the Peltier effect. For power generation, the thermoelectric efficiency $(\eta)$ is defined by combining the Carnot efficiency $\left(\Delta T / T_{\text {hot }}\right)$ and the figure of merit (ZT) as shown in the equation (Snyder and Toberer, 2008; Sootsman et al., 2009; Chen et al., 2003). 


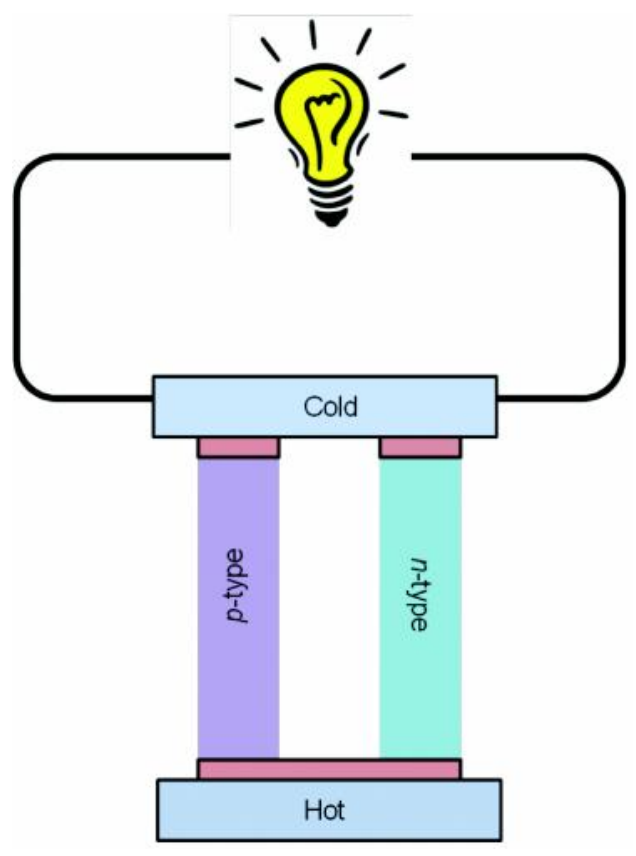

Fig. 1: Generic module diagram of a thermoelectric couple made of $n$-type and $p$-type materials in power generation mode

$$
\eta=\frac{\Delta T}{T_{\text {hot }}} \frac{\left(\sqrt{1+Z T_{\text {av }}}-1\right)}{\left(\sqrt{1+Z T_{\text {av }}}+\frac{T_{\text {cold }}}{T_{\text {hot }}}\right)}
$$

where $T_{\text {hot }}$ and $T_{\text {cold }}$ are the temperatures of the hot and cold ends in a thermoelectric device and $\Delta T$ their difference. This equation indicates that increasing efficiency requires both high $Z T$ values and a large temperature difference across the thermoelectric materials. Market-based thermoelectric devices currently available have a $Z T$ of $\sim 1$ and operate at an efficiency of only around 6-8\%. By increasing ZT by a factor of 4 , and depending on $\Delta T$, the predicted efficiency increases to $30 \%$, a highly attractive prospect. The challenge to create high ZT thermoelectric materials lies in achieving simultaneously high electronic conductivity $(\sigma)$, high Seebeck coefficient $(S)$ and low thermal conductivity $(\kappa)$ in the same solid. These properties define the dimensionless thermoelectric figure of merit $Z T=\left(S^{2} \sigma /\right.$ к) $T$, where $T$ is the temperature (Snyder and Toberer, 2008; Sootsman et al., 2009; Chen et al., 2003; DiSalvo, 1999; Trit, 2011; Li et al., 2010; Biswas et al., 2011; Biswas et al., 2012). The quantity $S^{2} \sigma$ is called the power factor and is the key to achieving high performance. A large power factor means that a large voltage and a high current are generated. The thermal conductivity $\kappa$ has a contribution from lattice vibrations, $\kappa_{\text {latt }}$, called the lattice thermal conductivity. Thus, $\kappa=\kappa_{\mathrm{el}}+\kappa_{\text {latt }}$, where $\kappa_{\mathrm{el}}$ is the electronic thermal conductivity. Naturally, the thermal conductivity must be low as a large temperature gradient must be maintained; a large thermal conductivity will short the thermal circuit.

The field of thermoelectrics presents an important challenge to synthetic chemists, physicists, as well as the materials scientists. The main challenge in this field is to develop highly efficient, stable, environment-friendly and inexpensive solid state materials. The discovery of new promising materials requires a combination of theoretical direction, intense chemical intuition, synthetic chemistry skill, materials processing, and good measurement expertise. This powerful combination can be effectively achieved by reaching across scientific disciplines.

\section{Why is Thermoelectrics Important to India?}

In the last decade, Indian economy has shown incredible growth. Steadily and slowly, India is gaining strategic importance globally owing to the impressive economic growth pattern and market attractiveness. With growing economy, there will be more energy consumption in the country. India is the world's 5th largest energy consumer accounting for about $4.1 \%$ of the world's total annual energy consumption and moving fast enough to become the third largest consumer by 2025 after USA and China (Source: India Energy Book, 2012). The per capita energy consumption of India is 0.5 toe (tonnes of oil equivalent) as compared to the world average of 1.9 toe, and this indicates a high potential for energy consumption (Source, 2012). Fig. 2A shows the graph of total energy consumption by type for India in 2011 (Source: US Energy Information Administration, 2012). It can be seen that major percentage of the energy was consumed in terms of the use of coal, petroleum and gas. In general, main share of the electricity is generated from coal, hydro and nuclear fuel. On the 

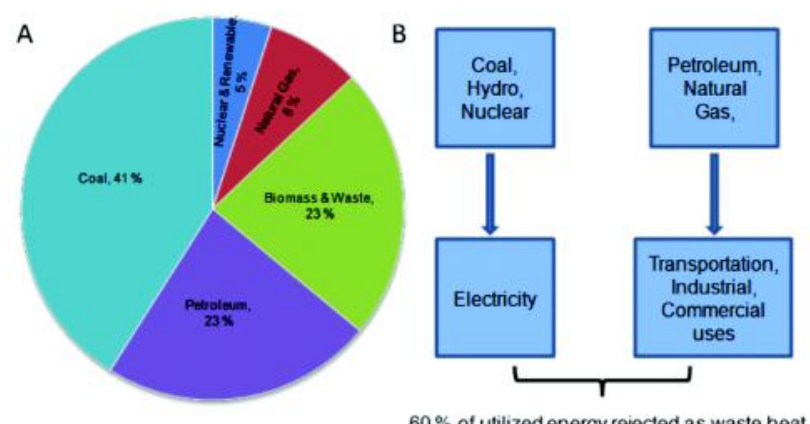

$60 \%$ of utilized energy rejected as waste heat

Fig. 2: (A) Total energy consumption by type for India in 2011. Source: U.S. Energy Information Administration, International Energy Statics, Indian Central Electricity Authority (2012). (B) Schematic shows partly the utilized energy being lost as waste heat

other hand, combustions of petroleum and gas are used to run the industrial and transportation sector in India. If we carefully observe that after the use of this enormous amount of energy in terms of electricity or combustion process, $\sim 60 \%$ of the utilized energy is being lost as waste heat (Fig. 2B) (Biswas et al., 2012). Could it be possible to make use of this untapped heat energy? The answer is yes, and 10$20 \%$ conversion to the useful form can have significant impact on overall energy. Thermoelectric materials allow the direct conversion between thermal and electrical energy. Different sectors of application include automobiles, heavy trucks and vehicles, coal burning electric utilities, and nuclear reactor facilities. Anything that uses an internal combustion engine (moving or stationary) can use these thermoelectric materials to convert waste heat to electrical energy for enhanced energy-efficiency. Basic research and extensive academic-industrial collaboration are essential to improve the existing thermoelectric efficiencies further to make it usable for major applications.

\section{Criteria for a Good Thermoelectric Material}

Primary object to the field of thermoelectric materials is the need to optimize a variety of interdependent properties. To maximize the thermoelectric figure of merit $(Z T)$ of a material, a large Seebeck coefficient, high electrical conductivity, and low thermal conductivity are required. As these transport characteristics depend on interrelated material properties, a number of parameters need to be optimized to maximize $Z T$. As high $Z T$ requires high electrical conductivity but low thermal conductivity, the Wiedemann-Franz law reveals an inherent materials conflict for achieving high thermoelectric efficiency. Electrical part of the thermal conductivity $\left(\kappa_{\mathrm{el}}\right)$ is directly related to electrical conductivity $(\sigma)$ by the relation, $\kappa_{e}=L \sigma T$, where $L$ is the Lorenz number (Snyder and Toberer, 2008; Sootsman et al., 2009). Ideally, a good thermoelectric material should have low thermal conductivity (property of glass), high electrical conductivity (property of metal) and large Seebeck (property of semiconductor). Challenge lies in the field to optimize all these important properties to achieve high thermoelectric performances.

\section{Reduction of the Thermal Conductivity}

A successful approach to increase the $Z T$ value has been introduced to modify an already promising compound by introducing point defects through the synthesis of solid solutions. The solid solution provides an environment of the atomic mass fluctuation throughout the crystal lattice (disorder), which gives rise to strong phonon scattering and generally can lead to the significantly lower thermal conductivity and a high $Z T$ value. For example, solid solutions of $\mathrm{PbTe}_{1-\mathrm{x}} \mathrm{Se}_{\mathrm{x}}$ and $\mathrm{Pb}_{1-\mathrm{x}} \mathrm{Sn}_{\mathrm{x}} \mathrm{Te}$ have lower thermal conductivity than that of pure PbTe (see Fig. 3) (Kanatzidis, 2010). Nanoscale inclusions in bulk materials can dramatically suppress lattice thermal conductivity by scattering the longer mean free path

A

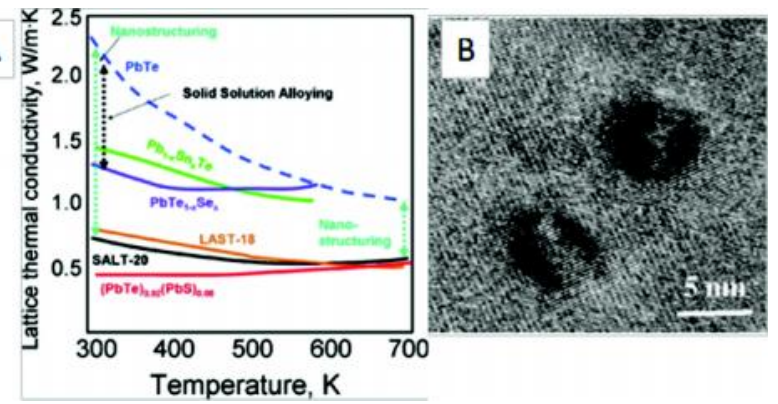

Fig. 3: (A) Lattice thermal conductivity as a function of temperature for various PbTe-based alloys and nanostructured samples. (B) High resolution transmission electron microscopy of a LAST-m sample. Source: Kanatzidis (2010) 
heat-carrying phonons, as in $\mathrm{AgPb}_{\mathrm{m}} \mathrm{SbTe}_{\mathrm{m}+2}$ (LASTm) (Fig. 3), which resulted in high ZT of 1.8 (Hsu et al., 2004). Calculations predict that a wide size distribution of nanoparticles is preferable since it can effectively scatter different phonon modes and reduce thermal conductivity. However, the power factor is also reduced because the nanoprecipitates increase carrier scattering, which in turn unfavourably affects the carrier mobilities.

An interesting idea to achieve high ZT was proposed by Slack and is referred to as the "phonon glass electron crystal" (PGEC) approach (Slack, 1995). A PGEC material features cages in its crystal structure inside which massive atoms can reside. These big atoms are small enough relative to the cage to rattle. This situation produces a phonon damping that can result in significant reduction of the lattice thermal conductivity. In the PGEC picture, a glasslike thermal conductivity can in principle coexist with charge carriers of high mobility. The PGEC approach has inspired a significant amount of new research and has led to significant increases in $Z T$ for several compounds such as the multiple filled skutterudites (Shi et al., 2011).

Cubic $\mathrm{AgSbTe}_{2}$ and $\mathrm{AgBiSe}_{2}$ compounds are renowned for their intrinsically low $\kappa_{\text {lat }}$ due to the strong anharmonicity of the bonding arrangements in these compounds (Morelli et al., 2008). Recent theoretical and experimental studies on a series of cubic bulk $\mathrm{I}-\mathrm{V}-\mathrm{VI}_{2}$ compounds have shown that the lone pair on the group $\mathrm{V}$ element plays an important role in deforming the lattice vibration, which results in strong bond anharmonicity (Nielsen et al., 2012). Valence electronic configuration of $\mathrm{Bi} / \mathrm{Sb}$ is $n s^{2} n p^{3}$, where only $n p^{3}$ electrons are involved in the bond formation with chalcogen valence electrons while the beguiling $n s^{2}$ electrons of $\mathrm{Bi} / \mathrm{Sb}$ form a lone pair. The origin of strong anharmonicity in $\mathrm{Bi} / \mathrm{Sb}-\mathrm{X}(\mathrm{X}=\mathrm{S} / \mathrm{Se} /$ $\mathrm{Te}$ ) bond is the electrostatic repulsion between the stereochemically active lone pair of $\mathrm{Bi} / \mathrm{Sb}$ and the valence bonding charge of the chalcogen (Guin et al., 2013).

\section{Increment of the Power Factor}

The important challenges in current thermoelectric research are how to increase the thermoelectric power $(S)$ of a material without depressing the electrical conductivity $(\sigma)$ and to predict precisely which materials will have a high power factor $\left(S^{2} \sigma\right)$. Generally, the thermoelectric power and electrical conductivity change in opposite directions with doping (Sootsman et al., 2009) and thus there is a compromised set of values that must be achieved. Generally, multiple pockets in valence or conduction band extrema give rise to high Seebeck coefficient (Snyder and Toberer, 2008). When the system is highly doped, more valleys are populated, thus resulting in high power factor. Convergence of multiple charges carrying electronic band valleys has virtually no detrimental effects on the carrier mobility $(\mu)$. Multiple degenerate valleys (separate pockets of Fermi surface with the same energy) have the effect of producing large effective mass $\left(m^{*}\right)$ without explicitly reducing $\mu$ (Pei et al., 2012). Flat/broad valence band maximum also gives rise to high $m^{*}$ (Guin et al., 2013, 2014a, b), thus resulting in enhanced $S$ as:

$$
S=\frac{8 \pi^{2} k_{B}^{2}}{3 e h^{2}} m^{*} T\left(\frac{\pi}{3 n}\right)^{3 / 2},
$$

where $k_{B}$ is the Boltzmann constant, $e$ is the electron charge, $h$ is the Planck constant and $n$ is carrier concentration.

Boltzmann transport theory describes both electronic and thermal transport in the vast majority of solids. This theory provides a general understanding of the thermopower $(S)$ that is expressed by the Mott equation (Sootsman et al., 2009):

$$
\left[S=\frac{\pi}{3} \frac{k_{B} T}{e} \frac{d \ln \sigma(E)}{d E}\right]_{E=E_{f}},
$$

$\sigma(E)$ is the electronic conductivity determined as a function of the band filling of Fermi energy, $E_{F}$. If electronic scattering is independent of energy, then $\sigma(E)$ is just proportional to the density of states (DOS) at $E$. Fig. 4 shows two hypothetical electronic DOS diagrams; one in which the DOS varies rapidly near $E_{F}$, and the other in which it does not. Based on the above equation, the system in Fig. 4A with sharp 


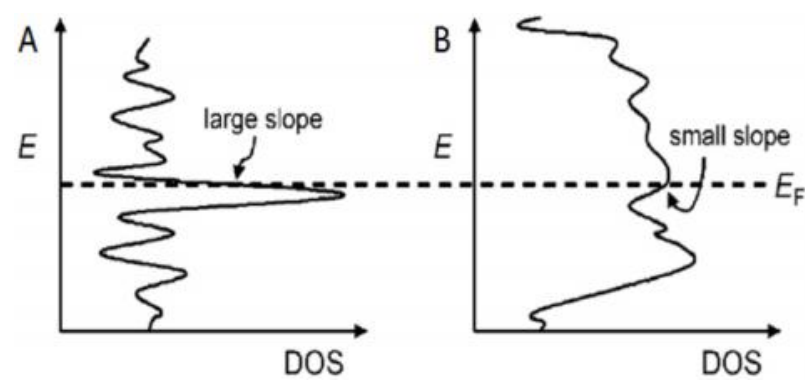

Fig. 4: Hypothetical density of state (DOS) with (A) a large slope and (B) a small slope near Fermi energy $\left(E_{F}\right)$. Source: Sootsman et al. (2009)

changing DOS is expected to have a larger thermoelectric power (Sootsman et al., 2009).

Thus, important parameters to consider when selecting or designing material systems are the band gap values, the shape and width of the bands near the Fermi level $\left(E_{\mathrm{F}}\right)$, and the carrier effective masses and carrier mobilities. The band gap is important because, in general, it is the temperature at which the ZT maximizes scales with band-gap size (Snyder and Toberer, 2008). This is because for a given band gap energy $\left(E_{\mathrm{g}}\right)$, there is a temperature at which thermally induced cross-gap carrier excitations occur to generate carriers of opposite sign which decrease the thermopower. Thus, for cooling applications, lower band-gap (0.1-0.2 eV) materials are best; whereas for high-temperature power generation, slightly larger band gaps (0.3-1 eV) are well-suited.

\section{State-of-the-art Thermoelectric Materials}

Various thermoelectric materials have been prepared and are present as device form in the mass market. My goal herein is to provide a brief overview about the most recent progress in thermoelectric materials. The thermoelectric figure of merit of the best recent thermoelectric materials is compared in Fig. 5 (Kanatzidis, 2010). Here onwards, a few of them are discussed case-by-case.

\section{Bismuth Chalcogenides}

$\mathrm{Bi}_{2} \mathrm{Te}_{3}$ is a narrow-gap semiconductor with an indirect gap of $\sim 0.15 \mathrm{eV}$ (Sootsman et al., 2009). $\mathrm{Bi}_{2} \mathrm{Te}_{3}$ crystallizes in the rhombohedral space group $R-3 m$ and the structure is made up of quintuple layers ( $\mathrm{Te} 1-$

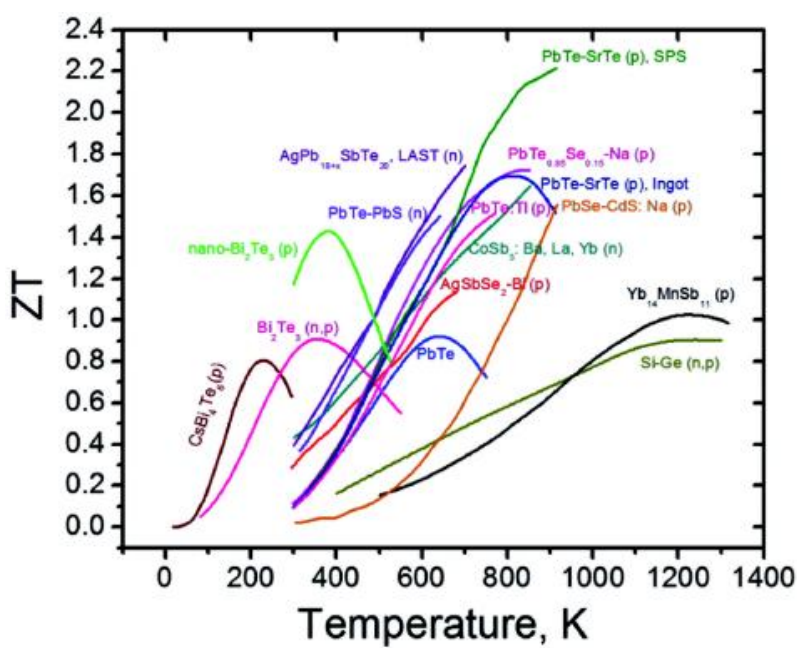

Fig. 5: Current state-of-the-art in bulk thermoelectric materials. Plot shows the temperature-dependent thermoelectric figure of merit $(Z T)$ vs $T$. Note: Data obtained from recent literature and Kanatzidis (2010)

Bi-Te2-Bi-Te1), stacked by van der Waals interactions along the $c$-axis in the unit cell (Fig. 6A). The stateof-the-art $\mathrm{Bi}_{2} \mathrm{Te}_{3}$ materials with $Z T=1$ are synthesized by alloying with $\mathrm{Sb}$ for $p$-type and $\mathrm{Se}$ for n-type materials. In actual devices, the $p$-type "legs" are
A

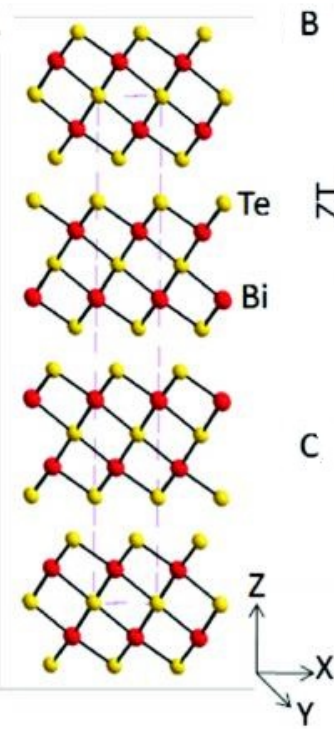

C
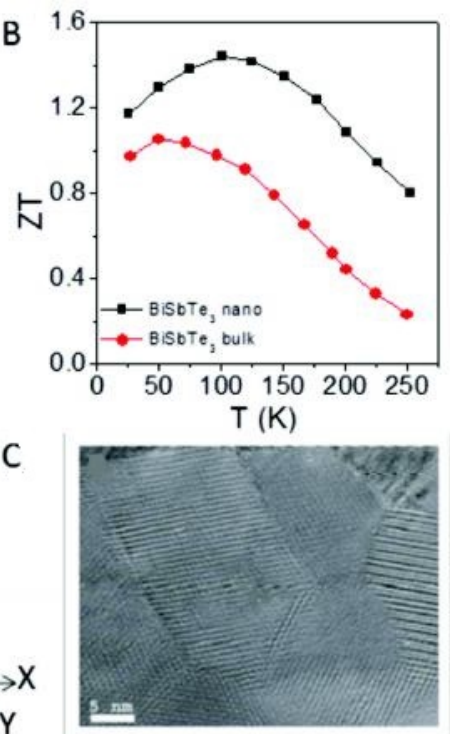

Fig. 6: (A) Layered crystal structure of $\mathrm{Bi}_{2} \mathrm{Te}_{3}$. (B) Temperature-dependent ZT of hot-pressed nanostructured and market-based ingot bismuth antimony telluride. (C) Transmission electron microscope (TEM) image showing nanocrystalline grain of high performance bismuth antimony telluride. Note: (B) and (C) from Poudel et al. (2008) 
generally hot-pressed and annealed pellets of $\mathrm{Bi}_{0.5} \mathrm{Sb}_{1.5} \mathrm{Te}_{3}$, which have good mechanical properties. The $n$-type counterpart is typically an ingot form of $\mathrm{Bi}_{2} \mathrm{Te}_{2.7} \mathrm{Se}_{0.3}$ grown by zone melting techniques. Melt spinning followed by spark plasma sintering (SPS) yielded p-type $\mathrm{Bi}_{2} \mathrm{Te}_{3}$ ingots with a $Z T$ value of 1.35 at $300 \mathrm{~K}$ (Tang et al., 2007). This material features $25 \mathrm{~nm}$ wide ribbons composed of nanostructured layers of $\mathrm{Bi}_{2} \mathrm{Te}_{3}$ crystals with $1 \mathrm{~nm}$ interplanar distance. The highest $Z T$ value for a bulk p-type $\mathrm{Bi}_{2} \mathrm{Te}_{3}$ material was reported recently (Poudel et al., 2008). The material with $Z T \sim 1.4$ at $100^{\circ} \mathrm{C}$ was prepared by ball milling followed by hot pressing (see Fig. 6B) (Poudel et al., 2008). The ZT enhancement for this system arises from reducing the lattice thermal conductivity while maintaining a comparable power factor to that of the bulk $p$-type $\mathrm{Bi}_{0.5} \mathrm{Sb}_{1.5} \mathrm{Te}_{3}$. This material is called "nanobulk" $\mathrm{Bi}_{2-x} \mathrm{Sb}_{x} \mathrm{Te}_{3}$ and it is a single-phase material composed of nanograins and micrograins mixed together (see Fig. 6C). Devices made of these nanostructured materials have shown superior thermoelectric efficiency than that of the devices from commercially available $p$-type $\mathrm{Bi}_{2} \mathrm{Te}_{3}$. Promising thermoelectric performance has been achieved in nanostructured $\mathrm{Bi}_{2} \mathrm{Te}_{3}, \mathrm{Sb}_{2} \mathrm{Te}_{3}$ and their alloys synthesized by bottom-up solution-based microwave-assisted synthesis (Mehta et al., 2012).

$\mathrm{CsBi}_{4} \mathrm{Te}_{6}$ is a promising material for lowtemperature thermoelectric applications (Chung et al., 2000). The presence of $\mathrm{Bi}-\mathrm{Bi}$ bonds in the structure is responsible for the very narrow energy gap $(\sim 0.08$ $\mathrm{eV})$, nearly half of that of $\mathrm{Bi}_{2} \mathrm{Te}_{3}$. The narrower band gap is responsible for the maximum $Z T$ value in $\mathrm{CsBi}_{4} \mathrm{Te}_{6}$ being at lower temperature than that of $\mathrm{Bi}_{2} \mathrm{Te}_{3}$. A ZT value of 0.8 at $225 \mathrm{~K}$ was obtained for $0.06 \% \mathrm{SbI}_{3}$-doped $\mathrm{CsBi}_{4} \mathrm{Te}_{6}$.

\section{Lead Chalcogenides}

$\mathrm{PbTe}$ is the champion thermoelectric material for midrange temperature $(600-800 \mathrm{~K})$ applications. It crystallizes in the $\mathrm{NaCl}$ crystal structure with $\mathrm{Pb}$ atoms occupying the cation sites and Te forming the anionic lattice. A band gap of $0.32 \mathrm{eV}$ allows it to be optimized for power-generation applications and can be doped in either $n$ - or $p$-type with appropriate dopants.

Significant enhancement of the Seebeck coefficient was achieved by introducing resonance level (sharp changes is density of states) in the valence band of PbTe by doping 2 mol\% thalium, which resulted in the doubling of $Z T$ to 1.5 at $773 \mathrm{~K}$ (Heremans et al., 2008). The $p$-type Na-doped $\mathrm{PbTe}_{1 \text { - }}$ ${ }_{x} \mathrm{Se}_{x}$ also exhibits high performance thermoelectric properties (ZT 1.8 at $850 \mathrm{~K}$ ) arising from convergence of the multiple valence bands (Pei et al., 2011).

Nanoscale inclusions in bulk materials can dramatically suppress the lattice thermal conductivity $\left(\kappa_{\text {latt }}\right)$ by scattering the longer wavelength heatcarrying phonons, as shown for the first time in $\mathrm{AgPb}_{\mathrm{m}} \mathrm{SbTe}_{\mathrm{m}+2}$ (Hsu et al., 2004). In all these cases, however, the power factor $\left(S^{2} \sigma\right)$ is also reduced because the nanoinclusions increase carrier scattering which in turn adversely affects the carrier mobilities. Recently, it was observed that by embedding endotaxial SrTe nanocrystals at a concentration as low as $2 \%$ in $p$-type bulk PbTe, the heat flow can be greatly inhibited without affecting the carrier mobility, thereby allowing a large power factor to be maintained (Biswas et al., 2011). The insensitivity of carrier scattering was attributed to valence band alignment of $\mathrm{SrTe}$ and $\mathrm{PbTe}$ allowing facile hole transport. The crystallographic alignment of $\mathrm{SrTe}$ and $\mathrm{PbTe}$ lattices and associated strain at interfaces decouples phonon and hole transport leading to a thermoelectric figure of merit of 1.7 at $~ 800 \mathrm{~K}$ (Biswas et al., 2011). Later, similar research has been extended to $\mathrm{PbTe}-\mathrm{MgTe} /$ $\mathrm{CaTe} / \mathrm{BaTe}$ (matrix-nanoprecipitate) system, where promising thermoelectric performances have also been achieved (Biswas, He et al., 2011; Ohta et al., 2012; Lo et al., 2012). Recently, Biswas and Kantzidis have demonstrated the substantial suppression of lattice thermal conductivity at high temperature in the $\mathrm{PbTe}-\mathrm{SrTe}$ system that leds to a record high $Z T$ of $\sim 2.2$ at $915 \mathrm{~K}$ in spark plasma sintered-processed samples (Biswas et al., 2012). This is the result of introducing phonon scattering at all-length scales in a hierarchical fashion from atomic scale doping and endotaxial nanostructuring to mesoscale grain boundary engineering (Fig. 7). With this new advance in the maximum $Z T$, average $Z T_{\text {avg }}$ values of $\sim 1.2$ and 
A
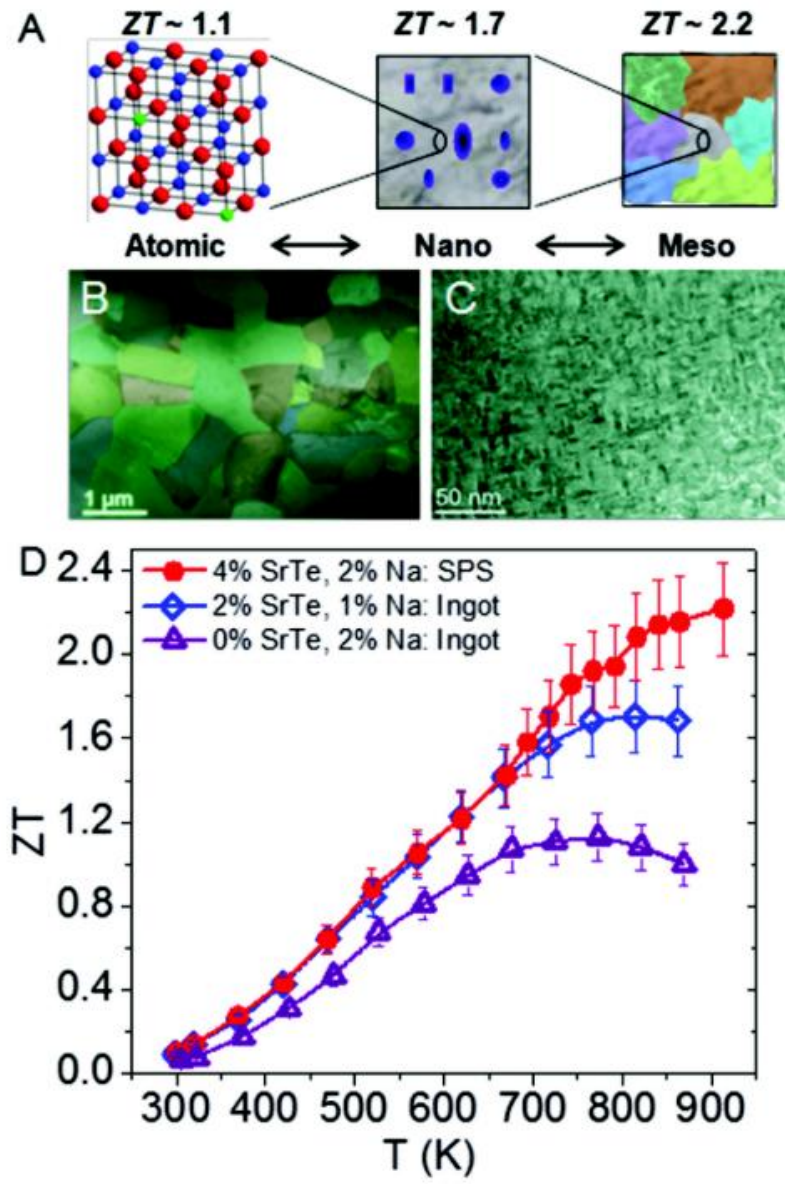

Fig. 7: (A) Maximum achievable $Z T$ values for the respective length scales: the atomic scale (alloy scattering), the nanoscale (PbTe matrix, grey; SrTe nanocrystals, blue) to the mesoscale (grain-boundary scattering). (B) and (C) TEM images show the micro and nanostructures in spark-plasma-sintered PbTe-SrTe doped with Na. (D) Temperature-dependent $Z T$ for an ingot (atomic scale), endotaxial nanostructuted PbTe (atomic plus nanoscale) and spark-plasma-sintered PbTe (atomic + nano + mesoscale). Source: Biswas et al. (2012)

$\sim 1.7$ were obtained for non-segmented and segmented thermoelectric devices, respectively (segmentation with BiSbTe, $Z T \sim 1.2$ at $350 \mathrm{~K}$ ). Considering a cold side temperature of $350 \mathrm{~K}$ and hot side temperature of $950 \mathrm{~K}$ for such devices, waste heat conversion efficiencies, respectively of $\sim 16.5 \%$ and $\sim 20 \%$ were predicted (Biswas et al., 2012).

\section{Silver Antimony Chalcogenides}

Fifty years earlier, Rosi (Rosi et al., 1961) recognized $\mathrm{AgSbTe}_{2}$ to be an efficient $p$-type thermoelectric material with $Z T$ of 1.3 at $720 \mathrm{~K}$. In the recent years, $\mathrm{AgSbTe}_{2}$ compound has been repeatedly studied to improve performance further by optimization carrier concentration through various doping. Interestingly, $\mathrm{AgSbTe}_{2}$ alloys with GeTe (TAGS) (Lee et al., 2014; Salvador et al., 2009; Zhang et al., 2013) and PbTe (LAST-m) (Hsu et al., 2004) showed extraordinary $Z T$ values $\sim 1.5$ at $750 \mathrm{~K}$ and $\sim 1.8$ at $800 \mathrm{~K}$, respectively. Recently, we have shown from India that enhanced electrical transport and ultra low thermal conductivity resulted in high thermoelectric performance of $\mathrm{Pb}$ or Bi doped bulk $p$-type $\mathrm{AgSbSe}_{2}$ which is Se analogue of $\mathrm{AgSbTe}_{2}$ (Guin et al., 2013). The maximum ZT achieved is 1.2 at $685 \mathrm{~K}$ for $2 \mathrm{~mol} \%$ Bi-doped sample, which is $190 \%$ higher than pristine $\mathrm{AgSbSe}_{2}$ sample (Fig. 8). With this advance in the maximum $Z T$ values of this Te-free material, we can expect an average $Z T$ value of $\sim 0.75$ (considering a hot side temperature of $700 \mathrm{~K}$ and cold side temperature of $350 \mathrm{~K}$ ), which is higher than leading metal selenide-based thermoelectric systems reported recently in literature (Fig. 8) (Guin et al., 2013). Considering a cold side temperature of $350 \mathrm{~K}$ and hot side $700 \mathrm{~K}$ for such devices, waste heat conversion efficiencies, respectively of $\sim 9 \%$ was predicted, which is comparable to market-based metal telluride devices. We have also shown that by introducing second phase nanostructures and proper carrier engineering, high ZT can be achieved in $\mathrm{AgSbSe}_{2}$ (Guin et al., 2014a, b).

\section{Skutterudites}

Skutterudites are a extremely promising class of compounds for thermoelectric power generation (Shi et al., 2011). They crystallize in the cubic $\mathrm{CoAs}_{3}-$ type structure with the space group Im-3. The structure is composed of eight-corner-shared $\mathrm{XY}_{6}$ (X=Co, Rh, Ir; Y=P, As, Sb) octahedra. Linked octahedra gives rise to a void at the centre of the $\left(\mathrm{XY}_{6}\right)_{8}$ cluster, where the void space occupies a bodycentered position of the cubic lattice. This void is large enough to accommodate large metal atoms to form filled skutterudites. Since the void-filling atoms can act as electron donors or acceptors, partially filling the void space of skutterudites could lead to an optimum electron concentration. At the same time, 

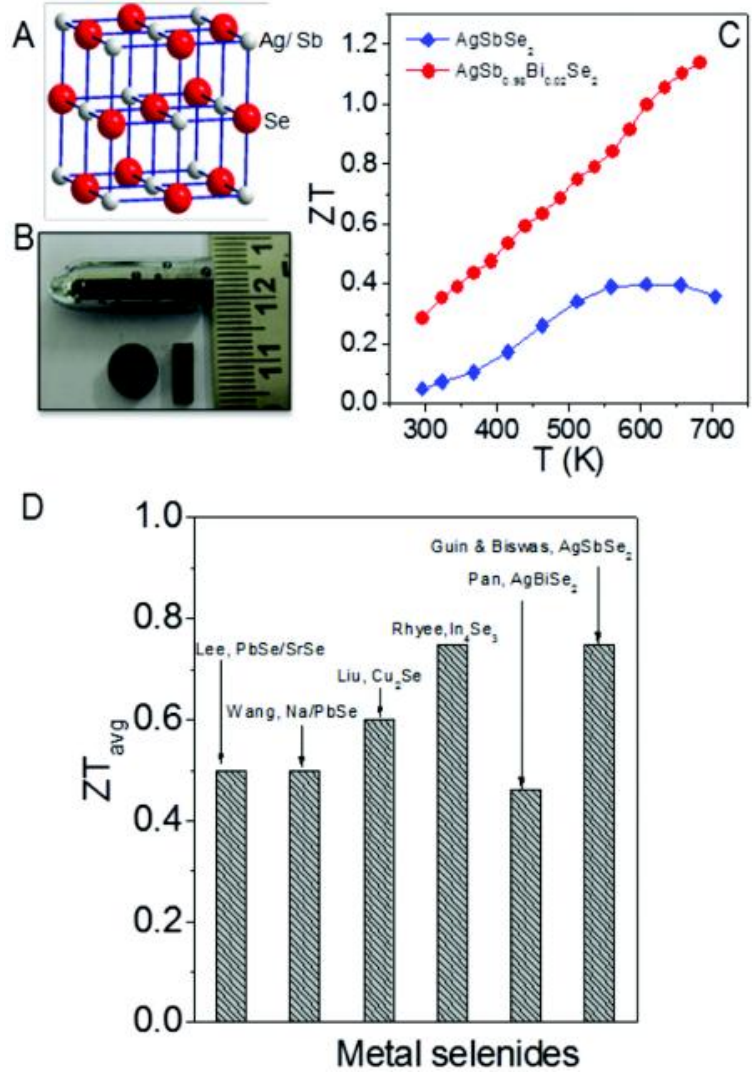

Fig. 8: (A) Crystal structure of cubic rocksalt $\mathrm{AgSbSe}_{2}$ with disordered Ag/Sb positions. (B) Photograph of assynthesized ingot. Bar- and coin-shaped samples are used for electrical and thermal transport measurements, respectively. (C) Temperaturedependent $Z T$ of Bi-doped and pristine $\mathrm{AgSbSe}_{2}$. (D) Average $Z T$ of present $\mathrm{AgSb}_{0.98} \mathrm{Bi}_{0.02} \mathrm{Se}_{2}$ and leading metal selenides reported in recent literature, considering a hot side temperature of $690 \mathrm{~K}$ and cold side temperature of $350 \mathrm{~K}$. Source: Guin et al. (2013)

these atoms can also act as strong phonon-scattering centres to greatly reduce the lattice thermal conductivity. The "rattling" effect of these void-filling atoms has resulted in improvements in the thermoelectric properties of skutterudite. High ZT values of partially filled skutterudites with a small amount of $\mathrm{Ni}$ doping for $\mathrm{Co}, \mathrm{Ba}_{0.30} \mathrm{Ni}_{0.05} \mathrm{Co}_{3.95} \mathrm{Sb}_{12}$ (ZT 1.25 at $900 \mathrm{~K})$ were reported (Tang et al., 2005). Further improvement in the ZT was achieved by filling up multiple atoms in the void of $\mathrm{CoSb}_{3}$ (Shi et al., 2011).

\section{Half-Heusler Compounds}

Another class of compounds of considerable interest as potential thermoelectric materials for hightemperature applications are the half-Heusler $(\mathrm{HH})$ compounds (Snyder and Toberer, 2008; Sootsman et $a l ., 2009)$, given by the composition MNiSn (M=Ti, $\mathrm{Hf}, \mathrm{Zr}$ ). HH phases have the MgAgAs crystal structure which consists of three filled interpenetrating face centered cubic sublattices and one vacant sublattice. The general formula is XYZ, where $\mathrm{X}$ and $\mathrm{Y}$ are transition metals and $\mathrm{Z}$ is a main-group element. Another advantage of these compounds is their high melting points of $1100-1300^{\circ} \mathrm{C}$ as well as their chemical stability with essentially zero sublimation at temperatures near $1000^{\circ} \mathrm{C}$. The Heusler intermetallic compounds with fully filled sublattices are metals (fullHeusler alloys), whereas the vacant $\mathrm{Ni}$ atom sites in half-Heusler compounds give rise to narrow bands resulting in d-orbital hybridization and substantial semiconducting character of the compounds. Notable progress was reported with $Z T \sim 0.7$ at $800 \mathrm{~K}$ for $n$ type $\mathrm{Zr}_{0.5} \mathrm{Hf}_{0.5} \mathrm{Ni}_{0.8} \mathrm{Pd}_{0.2} \mathrm{Sn}_{0.99} \mathrm{Sb}_{0.01}$ (Shen et al., 2001). Sakurada and Shutoh (2005) reported a $Z T$ value near 1.4 at $700 \mathrm{~K}$ for n-type $\left(\mathrm{Zr}_{0.5} \mathrm{Hf}_{0.5}\right)_{0.5} \mathrm{Ti}_{0.5} \mathrm{NiSn}_{1-\mathrm{y}} \mathrm{Sb}_{\mathrm{y}}$. A study by Indian researchers shows enhanced thermoelectric performance $(Z T \sim 1.1$ at $773 \mathrm{~K})$ in nanostructured $\mathrm{Zr}_{0.25} \mathrm{Hf}_{0.75} \mathrm{NiSn}$ was prepared by ball milling followed by spark plasma sintering (Bathula et al., 2012).

\section{Future Outlook and Conclusions}

The development of new materials and complex composites over the last 5-10 years has significantly increased the $Z T$ values. It has been occurred through better theoretical understanding, development of new synthesis techniques, and state-of-the-art measurements that the field has progressed so far and promises to move forward further. For a long time, it was thought that there was a practical barrier at $Z T=1$; however, new mechanisms for increasing the power factor and reducing the thermal conductivity in thermoelectric materials continue to emerge and increase the $Z T$ value. The newest generation of bulk materials has $Z T \sim 1.6-2.2$ at approximately 700-900 $\mathrm{K}$ (Biswas et al., 2011, 2012; Pei et al., 2011). It is hoped that $Z T \sim 3$ will soon be achieved, which will provide a new generation of thermoelectric power generators with thermoelectric efficiency of $\sim 25 \%$. 
Future efforts in understanding these mechanisms promise to increase the $Z T$ value further and enable more practical application. The discovery of advanced thermoelectrics poses a challenge to chemists, physicists, materials scientists and engineers.

Further reductions in the thermal conductivity alone may be sufficient to raise $Z T$ values to 2.5 ; however, to reach values of 3-3.5 or greater, we also need dramatic enhancement in the power factor. This can be achieved with innovation of some new and unexpected single-phase materials. What is needed now are new physical concepts on how the power factor can be enhanced 2-4-fold in the existing leading materials.

For realistic applications, the cost of power generation - as governed by material, manufacturing, and heat exchanger costs - is also a critical factor which is not captured in ZT alone (Yee et al., 2013). Among the high performance materials, $\mathrm{PbTe}$ is the most efficient for power generation application at high temperature, whereas $\mathrm{Bi}_{2} \mathrm{Te}_{3}$-based materials are well-known for refrigeration near room temperature. These leading high performance materials are mainly

\section{References}

Bathula S, Jayasimhadri M, Singh N, Srivastava A K, Pulikkotil J, Dhar A and Bhudhani R C (2012) Enhanced thermoelectric figure-of-merit in spark plasma sintered nanostructured n-type SiGe alloys Appl Phys Lett 101 $2139021-5$

Biswas K, He J, Blum I D, Wu C I, Hogan T P, Seidman D N, Dravid V P and Kanatzidis M G (2012) High-performance bulk thermoelectrics with all-scale hierarchical architectures Nature 489 414-418

Biswas K, He J Q, Wang G, Lo S H, Uher C, Dravid V P and Kanatzidis M G (2011) High thermoelectric figure of merit in nanostructured p-type PbTe-MTe $(\mathrm{M}=\mathrm{Ca}, \mathrm{Ba})$ Energy Environ Sci 4 4675-4684

Biswas K, He J, Zhang Q, Wang G, Uher C, Dravid V P and Kanatzidis M G (2011) Strained endotaxial nanostructures with high thermoelectric figure of merit Nat Chem 3 160166

Biswas K, Zhao L-D and Kanatzidis M G (2012) Tellurium-free thermoelectric: The anisotropic n-type semiconductor based on tellurium which is enormously scarce in the earth's crust (Hu et al., 2008). Hence, the cost of Te is likely to rise sharply if Te-based thermoelectric materials reach the mass markets. Therefore, it would be desirable to develop alternative materials which minimize the use of rare and toxic elements such as $\mathrm{Te}$ and involve cheaper and abundant elements. $\mathrm{S}$ and $\mathrm{Se}$ are much more earth abundant and less expensive than Te. Highly promising thermoelectric performance was observed in optimized bulk $\mathrm{PbS}$ (Zhao et al., 2012), PbSe (Zhao et al., 2013) and $\mathrm{Bi}_{2} \mathrm{~S}_{3}$ (Biswas, Zhao et al., 2012), which can replace expensive $\mathrm{PbTe}$ and $\mathrm{Bi}_{2} \mathrm{Te}_{3}$ based thermoelectric materials. We have also observed high thermoelectric performance in Te-free bulk $\mathrm{AgSbSe}_{2}$ (Guin et al., 2013, 2014a, b).

\section{Acknowledgement}

KB greatly appreciates the support of DST Ramanujan Fellowship, New Chemistry Unit and Sheikh Saqr Laboratory. I apologize in advance to all the investigators whose research could not be cited owing to the requirement of brevity.

$\mathrm{Bi}_{2} \mathrm{~S}_{3}$ Adv Energy Mater 2 634-638

Chen G, Dresselhaus M S, Dresselhaus G, Fleurial J P and Caillat $\mathrm{T}$ (2003) Recent developments in thermoelectric materials Int Mater Rev 48 45-66

Chung D Y, Hogan T, Brazis P, Rocci-Lane M, Kannewurf C, Bastea M, Uher C and Kanatzidis M G (2000) $\mathrm{CsBi}_{4} \mathrm{Te}_{6}$ : A high-performance thermoelectric material for lowtemperature applications Science 287 1024-1027

DiSalvo F J (1999) Thermoelectric cooling and power generation Science 285 703-706

Guin S N and Biswas K (2013) Cation disorder and bond anharmonicity optimize the thermoelectric properties in kinetically stabilized rocksalt $\mathrm{AgBiS}_{2}$ nanocrystals $\mathrm{Chem}$ Mater 25 (15) 3225-3231

Guin S N, Chatterjee A and Biswas K (2014a) Enhanced thermoelectric performance in p-type $\mathrm{AgSbSe}_{2}$ by $\mathrm{Cd}$ doping RSC Adv 4 11811-11815

Guin S N, Chatterjee A, Negi D S, Datta R and Biswas K (2013) High thermoelectric performance in tellurium free p-type $\mathrm{AgSbSe}_{2}$ Energy Environ Sci 6 2603-2608 
Guin S N, Negi D S, Datta R and Biswas K (2014b) Nanostructuring, carrier engineering and bond anharmonicity synergistically boost the thermoelectric performance of p-type $\mathrm{AgSbSe}_{2}-\mathrm{ZnSe} J$ Mater Chem A 2 4324-4331

Heremans J P, Jovovic V, Toberer E S, Saramat A, Kurosaki K, Charoenphakdee A, Yamanaka S and Snyder G J (2008) Enhancement of thermoelectric efficiency in PbTe by distortion of the electronic density of states Science $\mathbf{3 2 1}$ 554-557

$\mathrm{Hu}, \mathrm{Z}$ and Gao C S (2008) Upper crustal abundances of trace elements: A revision and update Chem Geol 253 205-221

Hsu K F, Loo S, Guo F, Chen W, Dyck J S, Uher C, Hogan T, Polychroniadis E K and Kanatzidis M G (2004) Cubic $\mathrm{AgPb}_{\mathrm{m}} \mathrm{SbTe}_{2+\mathrm{m}}$ : Bulk thermoelectric materials with high figure of merit Science $\mathbf{3 0 3} 818-821$

Kanatzidis M G (2010) Nanostructured thermoelectrics: The new paradigm? Chem Mater 22 648-659

Lee J K, Oh M W, Kim B S, Min B K, Lee H W and Park S D (2014) Influence of $\mathrm{Mn}$ on crystal structure and thermoelectric properties of GeTe compounds Electron Mater Lett 10 813-817

Li J -F, Liu W -S, Zhao L-D and Zhou M (2010) High-performance nanostructured thermoelectric materials NPG Asia Mater 2 152-158

Lo S-H, He J Q, Biswas K, Kanatzidis M G and Dravid V P (2012) Phonon scattering and thermal conductivity in $\mathrm{p}$ type nanostructured PbTe-BaTe bulk thermoelectric materials Adv Func Mater 22 5175-5185

Mehta R J, Zhang Y, Karthik C, Singh B, Siegel R W, Tasciuc T B and Ramanath G (2012) A new class of doped nanobulk high-figure-of-merit thermoelectrics by scalable bottomup assembly Nat Mater 11 233-240

Morelli D T, Jovovic V and Heremans J P (2008) Intrinsically minimal thermal conductivity in cubic $\mathrm{I}-\mathrm{V}-\mathrm{VI}_{2}$ semiconductors Phys Rev Lett 101035901 1-4

Nielsen M D, Ozolins V and Heremans J P (2012) Lone pair electrons minimize lattice thermal conductivity Energy Environ Sci 6 570-578

Ohta M, Biswas K, Lo S-H, He J, Chung D -Y, Dravid V P and Kanatzidis M G (2012) Enhancement of thermoelectric figure of merit by the insertion of MgTe nanostructures in p-type $\mathrm{PbTe}$ doped with $\mathrm{Na}_{2} \mathrm{Te}$ Adv Energy Mater 2 11171123

Pei Y, Shi X, La Londe A, Wang H, Chen L and Snyder G J (2011) Convergence of electronic bands for high performance bulk thermoelectric Nature 473 66-69
Pei Y, Wang H and Snyder G J (2012) Band engineering of thermoelectric materials Adv Mater 24 6125-6135

Poudel B, Hao Q, Ma Y, Lan Y, Minnich A, Yu B, Yan X, Wang D, Muto A, Vashaee D, Chen X, Liu J, Dresselhaus M. S, Chen G and Ren Z (2008) High-thermoelectric performance of nanostructured bismuth antimony telluride bulk alloys Science 320 634-638

Rosi F D, Hockings E F and Lindenblad N E (1961) Semiconducting materials for thermoelectric power generation RCA Rev 22 82-121

Sakurada S and Shutoh N (2005) Effect of Ti substitution on the thermoelectric properties of ( $\mathrm{Zr}, \mathrm{Hf}) \mathrm{NiSn}$ half-Heusler compounds Appl Phys Lett 86082105 1-3

Salvador J R, Yang J, Shi X, Wang H and Wereszczak AA (2009) Transport and mechanical property evaluation of $(\mathrm{AgSbTe})_{1-\mathrm{x}}(\mathrm{GeTe})_{\mathrm{x}}(\mathrm{x}=0.80,0.82,0.85,0.87,0.90) \mathrm{J}$ Solid State Chem 182 2088-2095

Shen Q, Chen L, Goto T, Hirai T, Yang J, Meisner G?P and Uher C (2001) Effects of partial substitution of Ni by Pd on the thermoelectric properties of ZrNiSn-based half-Heusler compounds Appl Phys Lett 79 4165-4167

Shi X, Yang J, Salvador J R, Chi M, Cho J Y, Wang H, Bai S, Yang J, Zhang W and Chen L (2011) Multiple-filled skutterudites: High thermoelectric figure of merit through separately optimizing electrical and thermal transports $J$ Am Chem Soc 133 7837-7846

Slack G A (1995) Title In: CRC Hand Book of Thermoelectrics (Ed: Rowe D M) pp 407-440, CRC Press, Boca Raton

Snyder G J and Toberer E S (2008) Complex thermoelectric materials Nat Mater 7 105-114

Sootsman J, Chung D Y and Kanatzidis M G (2009) New and old concepts in thermoelectric materials Angew Chem Int Ed 48 8616-8639

Source: India Energy Book 2012, World Energy Council

Source: U.S. Energy Information Administration 2012, International Energy Statics, Indian Central Electricity Authority

Tang X, Xie W, Li H, Zhao W and Zhang Q (2007) Preparation and thermoelectric transport properties of highperformance p-type $\mathrm{Bi}_{2} \mathrm{Te}_{3}$ with layered nanostructure Appl Phys Lett 90012102 1-3

Tang X, Zhang Q, Chen L, Goto T and Hirai T (2005) Synthesis and thermoelectric properties of p-type- and n-type-filled skutterudite $\mathrm{R}_{\mathrm{y}} \mathrm{M}_{\mathrm{x}} \mathrm{Co}_{4-\mathrm{x}} \mathrm{Sb}_{12}$ (R:Ce,Ba,Y;M:Fe,Ni) J Appl Phys 97093712 1-10

Tritt T M (2011) Thermoelectric phenomena, materials, and applications Annu Rev Mater Res 41 433-448 
Yee S K, LeBlanc S, Goodson K E and Dames C (2013) \$ per W metrics for thermoelectric power generation: beyond ZT Energy Environ Sci 6 2561-2571

Zhang L, Wang W, Ren B and Guo J The effect of adding nano$\mathrm{Bi}_{2} \mathrm{Te}_{3}$ on properties of $\mathrm{GeTe}$-based thermoelectric material J. Electron Mater 201342 1303-1306

Zhao L-D, He J, Hao S, Wu C-I, Hogan T P, Wolverton C, Dravid V P and Kanatzidis M G (2012) Raising the thermoelectric performance of $\mathrm{p}$-Type $\mathrm{PbS}$ with endotaxial nanostructuring and valence-band offset engineering using CdS and ZnS J Am Chem Soc 134 16327-16336

Zhao L-D, Hao S, Lo S H, Wu C-I, Zhou X, Lee Y, Li H, Biswas K, Hogan T P, Uher C, Wolverton C, Dravid V P and Kanatzidis M G (2013) High thermoelectric performance via hierarchical compositionally alloyed nanostructures $J$ Am Chem Soc 135 7364-7370. 